\title{
MoReeSC : a framework for the simulation and analysis of sound production in reed and brass instruments
}

\author{
F. Silva, Ch. Vergez, Ph. Guillemain, J. Kergomard* \\ LMA, CNRS, UPR 7051, Aix-Marseille Univ., Centrale Marseille \\ F-13402 Marseille cedex 20, France. \\ V. Debut ${ }^{\dagger}$ \\ Applied Dynamics Laboratory, Campus Tecnológico e Nuclear, \\ Instituto Superior Técnico/Universidade Técnica de Lisboa, \\ 2686-953 Sacavem, Portugal.
}

July 15, 2013

\begin{abstract}
This paper presents a free and open-source numerical framework for the simulation and the analysis of the sound production in reed and brass instruments. This tool is developed using the freely distributed Python language and libraries, making it available for acoustics student, engineers and researchers involved in musical acoustics. It relies on the modal expansion of the acoustic resonator (the bore of the instrument), the dynamics of the valve (the cane reed or the lips) and of the jet, to provide a compact continuous-time formulation of the sound production mechanism, modelling the bore as a series association of Helmholtz resonators. The computation of the self-sustained oscillations is controlled by time-varying parameters, including the mouth pressure and the player's embouchure, but the reed and acoustic resonator are also able to evolve during the simulation in order to allow the investigation of transient or non-stationary phenomena. Some examples are given (code is provided within the framework) to show the main features of this tool, such as the ability to handle bifurcations, like oscillation onset or change of regime, and to simulate musical effects.
\end{abstract}

\section{Introduction}

One of the main goals of musical acoustics is to provide models and investigation methods to improve our understanding of the behaviour of musical instruments, with application to sound synthesis and assistance to instrument makers. In the case of woodwind instruments, since the bore

\footnotetext{
* $\{$ silva, vergez, guillemain, kergomard $\}$ @lma.cnrs-mrs.fr

† vincentdebut@itn.pt
}

is primarily responsible for the intonation and timbre [1] many contributions studied a linear model of resonator and its various resonances, leading to results on the playing frequencies and some aspects of the clean intonation and tone color. Knowledge about sound production mechanism has also improved with models including the intrinsic non-linear coupling with the excitation. Frequency-domain representations have been widely used to investigate steady-state regimes, either static solutions [2] or periodic oscillations (see, e.g., the harmonic balance calculations $[3,4,5,6]$ ). Worman [7], extended by Kergomard et al. [8] and Ricaud et al. [5], enhanced the distribution of the energy among the harmonics of the periodic oscillation, and, in the clarinet case, the importance of even harmonics relatively to odd ones. However descriptors obtained by steady-state regime observations do not fully account for the characterization of the woodwind musicality. Transients are also necessary in the sense that they carry information useful to listener to identify the instrument and to perceive the musician intention.

In addition to signal-based synthesis schemes, alternative approaches rely on the use of physical causes instead of the direct computation of the effects. The underlying hypothesis is that the finer the modelling of physics, the closer the behaviours of the natural and the virtual instruments, and the more natural the synthesized sound, within the limits of the human perception. The control parameters of such physics-based synthesis methods are chosen to be close to the way the musician interacts with the real instrument. Hence the control of this kind of tool has a learning curve similar to that of the real world case. From the scientific point of view, time-domain simulations are helpful in understanding the relation between variation in control parameters and characteristics of the resulting sound. 
For a given set of behaviour laws describing physical phenomena, various representations are possible which lead to many ways to find a suitable solution. Refs. [9, 10] provide overviews of the implementations of the physical modelling of musical instruments. The source-filter approach can be used in voice synthesis, the characteristics of the source being related to the glottal flow, and the filter mimicking the transfer function of the vocal tract. However, in wind instruments a stronger interaction forces a consideration of the feedback from the resonator. When considering problems involving wave propagation in bores or strings for example, coupled partial derivatives equations (PDE) arise. They may be approximated by using some numerical schemes like finite differences in space and/or time $[11,12]$, or they may be reduced to lumped models [13]. Other methods rely on modelling propagation as delays of wave variables, the other phenomena being treated as local filters [14]. When non-linearities are assumed to be limited to the excitation part, the problem can be stated using quantities defined at the non-linearities. In the assumption of linear acoustics, a resonator can be fully described by its reflection function at the entrance of the bore (or equivalently by its input impedance), numerically reduced to a difference equation (as in the Digital Waveguide Modelling [15], or in the manner of Guillemain et al. [16]) or a MultiConvolution Algorithm [17]. Instead of trying to describe all the peculiarities of the possibly complex shaped bore leading to high-order digital filters, one can aim to describe the resulting input impedance. Gazengel et al. [18] provide an interesting insight into the process of using measured impedances in order to estimate the reflection function that can simulate the behaviour of the acoustic resonator as seen by the excitation. However, this method can lead to high computational cost due to the broad support of the reflection function for non-trivial geometries.

The aim of this paper is to present a calculation of selfsustained oscillations in time domain based on the modal decomposition of the bore input impedance, publicly distributed as MoReeSC (MOdal Resonator-rEEd interaction Simulation Code). Modal analysis is widely used in musical acoustics to analyse and reproduce the vibration of complex vibrating structures, but few applications have been presented for self-sustained instruments ([19] for the study of the bowed string, [20] for sound synthesis, and [21] for a dynamical system approach). The expansion of the input impedance as an association of single d.o.f resonators replaces the convolution with the reflection function by the resolution of a set of order-2 ordinary differential equations.

The outline is as follows: the next section (Sec. 2) presents the model of a reed instrument (brass or woodwinds). Focus is then ( $\mathrm{Sec}$. 3) given to the control over the numerical implementation of the model and its time-domain resolution. Sec. 4 is devoted to the results of calculations and the discussion of the behaviour of the coupled system for various interesting cases like the role of reed dynamics on attacks and transient results for brass instruments changing the lip reed and the bore characteristics. It will also consider more technical aspects such as the contents of the different components of the input pressure derived from the modal decomposition of the impedance and the influence of the solver used in the time-domain simulations. Then Sec. 5 lists known limitations, before discussions and perspectives (Sec. 6).

\section{The core of the framework}

\subsection{The underlying modelling}

Since the pioneering work of Helmholtz [22], a wide variety of musical instruments are considered as self-sustained oscillators, and their behaviour can be explained by the mutual coupling of a resonator and an exciter. With a control theory representation, a physical quantity (flow rate, stress, etc...) produced by the excitation acts as a source for waves in the resonator and modifies its state. This behaviour implies a reaction of the resonator on the exciter and, under particular conditions, an oscillation can emerge. For a large class of instruments, the interaction between two parts can be considered localised with respect to the involved wavelengths (contact between the bow and a violin string, jet at the entrance of reed instruments and brasses). In this way it is possible to take advantage of this property: if the subsystems are linear and the non-linearities are localised at a single point, it is possible to couple the transfer functions representing the resonator and the excitation by a non-linear function $[23,24]$. While the principles that follow can be transposed to other instruments like bowed bars and strings, focus is hereafter given to the reed-like instruments (single reed, double reed, lips, vocal folds). The air column acts as the acoustic resonator, and the valve (hereafter also called reed) is the mechanic excitation. The coupling is done by the air flow modulated by the reed that enters the resonator.

\subsection{Transfer function of the reed}

In order to keep the framework general, the reed is not restricted to common models present in the literature, as the massless or a single d.o.f. reed [25]. A general class to represent linear systems has been adopted by relating the reed channel opening $h(t)$ to its driving term (the pressure difference $\Delta p(t)$ for single reeds) by a differential equation:

$$
\begin{aligned}
& \frac{d^{N} h}{d t^{N}}+a_{1} \frac{d^{N-1} h}{d t^{N-1}}+\ldots+a_{N-1} \frac{d h}{d t}+a_{N}\left(h(t)-h_{0}\right) \\
= & b_{0} \frac{d^{M} \Delta p}{d t^{M}}+b_{1} \frac{d^{M-1} \Delta p}{d t^{M-1}}+\ldots+b_{M-1} \frac{d \Delta p}{d t}+b_{M} \Delta p(t)
\end{aligned}
$$


with $M \leq N$, or, using the Laplace transform

$$
\frac{H(s)}{\Delta P(s)}=\frac{\sum_{m=0}^{M} b_{m} s^{M-m}}{\sum_{=0}^{N} a_{n} s^{N-n}}
$$

where $s$ is the Laplace variable (usually evaluated on the frequency axis $s=j \omega)$, and $a_{0}=1$. The partial state vector $X_{\mathrm{m}}$ for the reed is defined as follows:

$$
X_{\mathrm{m}}=\left(h(t), \frac{d h}{d t}, \ldots, \frac{d^{N-1} h}{d t^{N-1}}\right)^{T}
$$

and the observable canonical form of the state-space representation has been adopted

$$
\frac{d X_{\mathrm{m}}}{d t}=F\left(t, X_{\mathrm{m}}, h_{0}, \Delta p\right) \text { and } h(t)=C X_{\mathrm{m}}(t)
$$

where $F$ involves the coefficients $a_{n}(n \in[1, N])$, and $b_{n}(n \in[0, M])$, and $C$ is the row-vector $[1,0, \ldots 0]$. Using the canonical state space observer form (see e.g. Ref. [26], App. G), the implementation of this continuous-time formulation does not require knowing $\Delta p(t)$ at previous instant, neither one of its derivatives, even for $M \geq 1$.

For instance, the classical single d.o.f. reed model from Ref. [25] is handled by the partial vector state $X_{\mathrm{m}}=$ $\left(h(t), \frac{d h}{d t}\right)$ whose evolution is defined by

$$
\frac{d X_{\mathrm{m}}}{d t}=\left(\begin{array}{cc}
0 & 1 \\
-\omega_{r}^{2} & -q_{r} \omega_{r}
\end{array}\right) X_{\mathrm{m}}+\left(\begin{array}{c}
0 \\
\omega_{r}^{2}\left(H_{0}-\Delta p / K\right)
\end{array}\right)
$$

where $K, \omega_{r}$ and $q_{r}$ denote the reed stiffness, natural angular frequency and damping, respectively, and $H_{0}$ is the opening area at rest without pressure difference.

This simple formulation may appear to be insufficient when the vibrations of the reed are not small. For instance the beating reed case requires the addition of a force due to the contact between the reed and the table according to Ref. [27], or to use variable coefficients $\omega_{r}$ and/or $q_{r}$ if the reed bends gradually on the table [28]. In the current implementation of the beating reed, an additional visco-elastic force appears when the channel closes, taking into account the elasticity of the table. The values used in the model may come from experimental characterisation, see e.g. [29] for cane reeds, or [30] for lip reeds.

\subsection{Modal expansion of the input impedance}

As stated in the introduction, an alternative implementation of the behaviour of the acoustic resonator is to parametrise its input impedance $Z_{e}(\omega)=P(\omega) / U(\omega)$, avoiding thus a detailed modelling of the whole bore or a long-tailed impulse response (or reflection function), in order to minimise the computational cost. The modal expansion of the input impedance leads to the expression of the input pressure $p(t)$ as a sum of components $p_{n}(t)$ :

$$
p(t)=\sum_{n} p_{n}(t)
$$

whose evolutions are related to the input flow $u(t)$ by order-2 differential equations, i.e. as a series association of Helmholtz resonators, from a physical point of view. Even if usual 2nd-order denominator polynomials are usable [31], a representation based on poles $\left(s_{n}\right)$ and residues $\left(C_{n}\right)$ removes the derivative of the input flow from the formulation:

$$
\begin{gathered}
\forall n \in \mathbb{Z}, \frac{d p_{n}}{d t}=C_{n} u(t)+s_{n} p_{n}(t) \\
Z_{e}(s)=\frac{P(s)}{U(s)}=\sum_{n \in \mathbb{Z}} \frac{C_{n}}{s-s_{n}}
\end{gathered}
$$

Poles and residues may be known analytically for idealized models, obtained from numerical identification, or resulting from spatial modal analysis with observation at the entrance of the bore:

$$
p(t)=\sum_{n} p_{n}(t) \varphi_{n}(x=0)
$$

with spatial modes $\varphi_{n}$ normalized such that $\varphi_{n}(x=0)=1$. Taking into account the damping, the modes may be complex (see e.g. Ref. [32]) resulting in complex poles and residues. Real poles are directly handled, whereas pairs of complex conjugate poles are dealt conjointly. In fact, if $s_{i}$ and $s_{j}$ are complex conjugates, both $p_{i}$ and $p_{j}$ are then a complex signal but, due to hermitian symmetry, they contribute altogether to the total pressure by the real quantity $2 \Re \mathfrak{e}\left(p_{i}(t)\right)$, so that only poles with non-negative imaginary part (i.e. related to non-negative eigenfrequencies) are to be considered:

$$
p(t)=\sum_{\Im \mathfrak{m}\left(s_{n}\right)>0} 2 \Re \mathfrak{e}\left(p_{n}(t)\right)+\sum_{\Im \mathfrak{m}\left(s_{n}\right)=0} p_{n}(t) .
$$

Within the control theory formalism, the partial state vector $X_{\mathrm{ac}}$ contains the components $p_{n}\left(n \in\left[1, N_{a}\right]\right)$ associated to poles with non-negative imaginary part and the observable $p$ can be evaluated using Eq. (10). The dynamics of the acoustic resonator is given by Eq. (7), or, equivalently, by

$$
\frac{d X_{\mathrm{ac}}}{d t}=\left(\begin{array}{c}
\frac{d p_{1}}{d t} \\
\vdots \\
\frac{d p_{N_{a}}}{d t}
\end{array}\right)=\left(\begin{array}{c}
C_{1} \\
\vdots \\
C_{N_{a}}
\end{array}\right) u(t)+\operatorname{diag}\left(s_{n}\right) X_{\mathrm{ac}} .
$$

An important question about the modal expansion of physical systems is the order retained, i.e. how the truncation of the possibly infinite expansion is performed. It is an active area of research (see e.g. $[33,34]$ ), and some correction term have been proposed for the case of cylindrical bores [35]. While suitable for resonators with no instantaneous response in reflection function, further work needs to be done when the geometry at the entrance of the acoustic resonator produces an instantaneous reflection as in the case of the saxophone and the brasses.

Another feature is that the input impedance may include both upstream and downstream airways as a series association of resonators with the same airflow. Considering 
suitable conventions for the bore impedance and the vocal tract (due to the direction of flow), $p(t)$ would then denote the acoustic pressure difference between the two faces of the reed, and $P_{m}$ the quasi-static component of the mouth pressure.

\subsection{The aero-elastic coupling}

As noted by Hirschberg [36], in the case of clarinet-like instruments, the control of the flow rate by the reed position is due to the existence of a turbulent jet. Indeed, a jet is supposed to form in the embouchure (pressure $p_{j e t}$ ) after the flow separation from the walls, at the end of the (very short) reed channel. Neglecting the air flow velocity in the mouth compared to the jet velocity $v_{j e t}$, the Bernoulli theorem applied between the mouth (pressure $P_{m}$ ) and the reed channel leads to $1 / 2 \rho v_{j e t}^{2}=P_{m}-p_{j e t}$. Assuming a rectangular aperture of width $W$ and height $h(t)$ driven by the reed displacement, the flow rate $u$ across the reed channel can be expressed as follows:

$$
u(t)=W h(t) \sqrt{\frac{2\left(P_{m}-p_{j e t}\right)}{\rho}},
$$

with $\rho$ the mass density. Since the cross section of the embouchure is large compared to the cross section of the reed channel, it can be supposed that all the kinetic energy of the jet is dissipated through turbulence with no pressure recovery (as in the case of a free jet). Therefore, the pressure in the jet $p_{j e t}$ is (assuming pressure continuity) the acoustic pressure $p(t)$ imposed by the resonator response to the incoming flow rate $u(t)$. Complementary overviews of the limits of this model are provided in Ref. [37, 38].

In the general case, we shall consider another partial state vector $X_{f}$ for the fluid dynamics. Since the expression (12) of the input flow is instantaneous, this is a mere observable that can be retrieved from $h(t)$ and $p(t)$, i.e. from $X_{\mathrm{m}}$ and $X_{\text {ac }}$. Obviously, a more complex flow equation can lead to an non-empty partial state vector $X_{f}$. For instance, the Fant's model can be handled by considering the simple partial state vector $X_{\mathrm{f}}=(u)$ with the following dynamics:

$$
\frac{d X_{f}}{d t}=\frac{1}{\mathcal{L}}\left(P_{m}-p_{j e t}-\mathcal{R} u-\rho \frac{u^{2}(t)}{2 h^{2}(t)}\right)
$$

with $\mathcal{L}$ the glottis inductance and $\mathcal{R}$ representing the viscous effects at the constriction (see Ref. [39]). So that $\dot{X}_{f}(t)$ is a function of $X(t)$ and of the control parameters. Ref. [40] can be considered as well.

Another possibility allowed by the dynamical system formulation is to take into account the reed motion induced flow [41]. This latter may be related to the reed velocity, that is part of the mechanic partial state vector, so this feature adds no additional difficulty.

\subsection{The full system}

Denoting $X=\left(X_{\mathrm{ac}}, X_{\mathrm{m}}, X_{\mathrm{f}}\right)^{T}$ the complete state vector, the procedure providing the dynamics of the full system as a simple function $\dot{X}=f(X, t)$ is detailed in Algorithm 1 .

Algorithm 1 Function $\dot{X}=f(X, t)$

$t$ and $X$ are the inputs given by the solver

1: Estimate parameters at time $t$

2: Build pressure $p(t)$ from $X_{\text {ac }}$ (see Eq. (10))

3: Extract reed channel opening $h(t)$ from $X_{m}$

4: Expand flow rate $u(t)$ from $X_{f}$ or from Eq. (12)

At this point all terms in r.h.s are known.

5: Compute $\dot{X}_{\mathrm{ac}}$ from Eq. (11), $\dot{X}_{\mathrm{m}}$ from Eq. (4) and $\dot{X}_{f}$ (if non-empty).

The derivative $\dot{X}$ is returned to the solver to continue the trajectory in the state space.

As an example, considering the single mode acoustic resonator with natural angular frequency $\omega_{1}$, quality factor $Q_{1}$ and amplitude $Z_{1}$ :

$$
\frac{d^{2} p}{d t^{2}}+\frac{\omega_{1}}{Q_{1}} \frac{d p}{d t}+\omega_{1}^{2} p=\frac{Z_{1} \omega_{1}}{Q_{1}} \frac{d u}{d t},
$$

and the Taylor series expansion of the Bernoulli-based flow rate relationship up to order 3 with a massless reed $(N=$ $M=0$ in Eq. (1))

$$
u(t)=u_{0}+A p(t)+B p(t)^{2}+C p(t)^{3}
$$

(see Ref. [42] for details) lead to the Van der Pol oscillator:

$$
\begin{aligned}
& \frac{d^{2} p}{d t^{2}}+\frac{\omega_{1}}{Q_{1}} \frac{d p}{d t}\left[1-Z_{1}\left(A+2 B p(t)+3 C p(t)^{2}\right)\right] \\
& +\omega_{1}^{2} p(t)=0 .
\end{aligned}
$$

In this simple example, the state vector reduces to its acoustic part, i.e. $X_{\mathrm{m}}$ and $X_{\mathrm{f}}$ are empty so that $X=\left(p_{1}\right)$. Considering the pole $s_{1}$ and its residue $C_{1}$ :

$$
\left\{\begin{array}{l}
s_{1}=\frac{\omega_{1}}{2 Q_{1}}\left(-1+j \sqrt{4 Q_{1}^{2}-1}\right), \\
C_{1}=\frac{Z_{1} \omega_{1}}{Q_{1}}\left(1+\frac{j}{\sqrt{4 Q_{1}^{2}-1}}\right) .
\end{array}\right.
$$

Algorithm 1 then reduces to Algorithm 2.

\subsection{Time-varying control parameters}

The simulation of the sound production process can only lead to perceptively natural sounds if the time evolution of the parameters that are usually controlled by the musician are not oversimplified [43]. On single reed instruments, the 


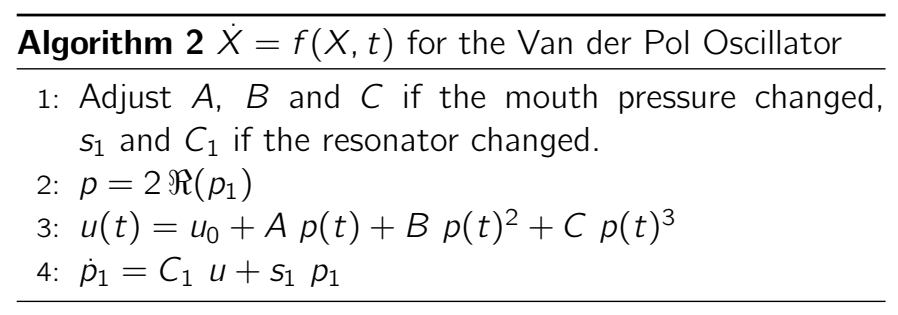

fingering (i.e. the bore impedance), the mouth pressure and the equilibrium reed channel opening are the main parameters that need to be controllable. However, in lip-reed instruments or in order to achieve some non-trivial effects, the reed's dynamics coefficients should be adapted along the duration of the phrase.

Such a feature is available in the MoReeSC software: every coefficient of the reed model, of the impedance and of the aero-elastic coupling can be specified as a function of the time only. In accordance with the continuous time simulation requirement, these functions need to be (possibly piecewise) parametrised curves: either a mere constant value, a linear interpolation, a Bézier curve or a B-spline built from a list of (instants,value) pairs. Measured signals can be used too, through B-splines interpolation and/or approximation. These profiles are then transformed without any approximation into a sequence of Bézier curves for computational efficiency.

The implementation thus allows and eases the investigation of dynamic phenomena like attacks, legato and slurred transients, as it will be shown below.

\section{Time-domain resolution}

The description of the behaviour of the three parts using a set of explicit ordinary differential equations allows for the use of various integrators. According to the purpose and requirements of the simulation, either fixed or variable stepsize methods are available. The former, derived from the Euler method, ensure speed and are intended for embedded or low performances devices. They are however not suitable with strongly non-linear phenomena (like beating reed) or with very short-time transients, which would require to increase the sampling frequency (this increase being worthless most of time), or to develop case-specific adaptation for each kind of singularity.

Another solution providing accurate results for stiff problems is based on the ability to change the step-size depending on the local properties of the dynamical system, and switching when possible between predictor-corrector methods and (implicit) backward differentiation formula with small step-size. The default choices in the MoReeSC software bases on the Fortran implementations LSODA [44] and VODE [45] from the LLNL (Lawrence Livermore National Laboratory). To determine if the problem is stiff, they rely on the eigenvalues of the jacobian matrix $J(X, t)$ of the dynamical system, which is either estimated by the solver using finite differences or evaluated through a procedure similar to the Algorithm 1, with an equivalent cost. Furthermore, for the solvers derived from LSODE (the family of solvers including LSODA) and VODE, only an approximation of the jacobian matrix is needed, lowering this cost compared to the one of the exact evaluation.

Nevertheless, any Ordinary Differential Equations solver provided by the open-source software Scipy [46] or derivatives can be used. The modularity of the MoReeSC software eases the choice and switch of the integrator (even within a simulation) providing, amongst other possibilities, a way to investigate the stability and the approximation of finite-difference schemes. Simulation can be interrupted and continued transparently, increasing the usability for example to quickly determine if the oscillation conditions have been met, letting the user analyse the already computed signal and decide to continue the simulation.

Considering computational costs, they naturally depend on the solver used. In order to enable continuation of the simulation and fine-grained analysis of the results, the state vector is stored at each point of a fixed-step time vector (defining a pseudo sampling frequency $f_{s}$ for the simulation). The base memory requirement is then $N M$ where $N$ is the number of state variables (i.e. the number of ODEs), and $M$ the number of samples corresponding to the time range of the simulation. Moreover each ODE integrator may need internal work arrays to handle multistep methods. Currently, to our best knowledge, the worst case occurs using the variable step, variable-order implicit Adams-Moulton method (as possibly used in LSODA and VODE) with the highest implemented order (12) leading to an additional requirement of a real work array of length $22+17 \mathrm{~N}$ and an integer work array of length $20+N$, which is quite negligible comparing to the base memory requirement. Refer to the respective documentations of the advanced solvers [44, 45] for details, notably concerning numerical stability. From the user point of view, all these details are handled transparently by the MoReeSC software, even if technical information about intermediate step sizes, switches of the estimated stiffness, local truncation error and convergence tests can be output for advanced solvers.

The following time integrators are currently available:

Explicit Euler method: the bounded stability region requires small time steps. For usual audio sampling frequencies, this condition forbids the explicit single-step method, leading to instability and explosion of the computed solution. Practically, the sampling frequency can be increased (by a factor $M$ ), it is quite usual for the time step to be about $0.01 / f_{s}(M=100$ intermediate steps between samples at the usual audio sampling frequency $f_{s}=44.1 \mathrm{kHz}$ ). The result is then obtained discarding the intermediate steps, as in the following 
Runge-Kutta methods.

Explicit Runge-Kutta methods of order 5(4) and 8(5,3) perform adaptive step size to bound the local truncation error [47]. As in the Euler integrator, the intermediate steps used to obtain the high order are discarded.

VODE and LSODA are multistep methods that re-use previous steps to gain efficiency instead of computing and discarding intermediate steps. They are also variable-order and adaptive step size, and can use both implicit Adams-Moulton methods (AM) or Backward differentiation formulas (BDF) depending on whether the problem is locally stiff or not. LSODA has the advantage of automatically performing the switch between the two methods when stiffness has been detected.

\section{Results}

Physically-based synthesis allows a higher level control than signal-based one, the control parameters being in connection with the physical and geometrical characteristics of the real instrument and with the actual musical command. The virtual device thus behaves likewise the real one, requiring a similar practise. An illustration of this feature lies in the ability to produce a variety of sounds depending on the evolution in time of the control parameters.

\subsection{Single reed instruments}

\subsubsection{Regime selection by the reed damping}

As a first example, the influence of the damping of a single d.o.f. reed on the clarinet is investigated in Fig. 1. The simulation involves a cylindrical bore of length $30 \mathrm{~cm}$ and radius $7 \mathrm{~mm}$ (truncated to 12 modes), and a reed with effective stiffness $0.5 \mathrm{GPa} / \mathrm{m}^{2}$, a natural frequency of $1500 \mathrm{~Hz}$ and an equilibrium aperture of $4 \mathrm{~mm}^{2}$. The mouth pressure is a smooth step ( $\mathcal{C}^{2}$ continuous) from 0 to $780 \mathrm{~Pa}$, with an attack time of $0.5 \mathrm{~ms}$ starting at $t=5 \mathrm{~ms}$.

For damped reeds $\left(q_{r}=1.0\right)$, the acoustic resonator is able to impose an oscillation frequency mainly determined by the highest impedance peak. A mere cylinder taking into account visco-thermal losses has its first peak as the highest impedance resonance (approximately given by $c / 4 L$, with $c$ sound velocity in the free space, and $L$ the bore length). It is noticeable that a slightly less damped reed $\left(q_{r}=0.7\right)$ contributes stronger higher harmonics. For $q_{r}=0.4$, the oscillation switches after a short transient to the second register, i.e. an oscillation based on the second resonance of the bore. Previous works $[25,2,6]$ explained, relying on linear stability analysis, the selection of the operating mode according to the reed resonance damping. The present paper extends these studies beyond the oscillation threshold. When the reed damping decreases, the oscillation threshold related to the first acoustic resonance is passed over by the one of the second bore resonance (i.e. gets higher than), which in turns is subdued by the third one (see Ref. [48], Fig. 7.6). Time-domain simulation goes beyond the oscillation threshold and highlights the spectral enhancement in the vicinity of the reed resonance frequency, concerning both odd and even harmonics. For low damping $\left(q_{r}=0.2\right)$, the oscillation is mainly supported by the reed resonance, i.e. the third harmonic (which is very close to the reed natural frequency) carries the most part of the energy, the system acting thus as in an organ pipe.

\subsubsection{Spectral content of the components}

Before exposing another characteristic of the simulated sounds, it is necessary to emphasise the difference between the pressure components $P_{n}(\omega)$ and the Fourier series of the steady-state pressure signal. One component does not hold a single spectral component. Fig. 2 highlights the rich spectrum of each of the pressure components, showing the steady-state waveforms and their frequency-domain coun-

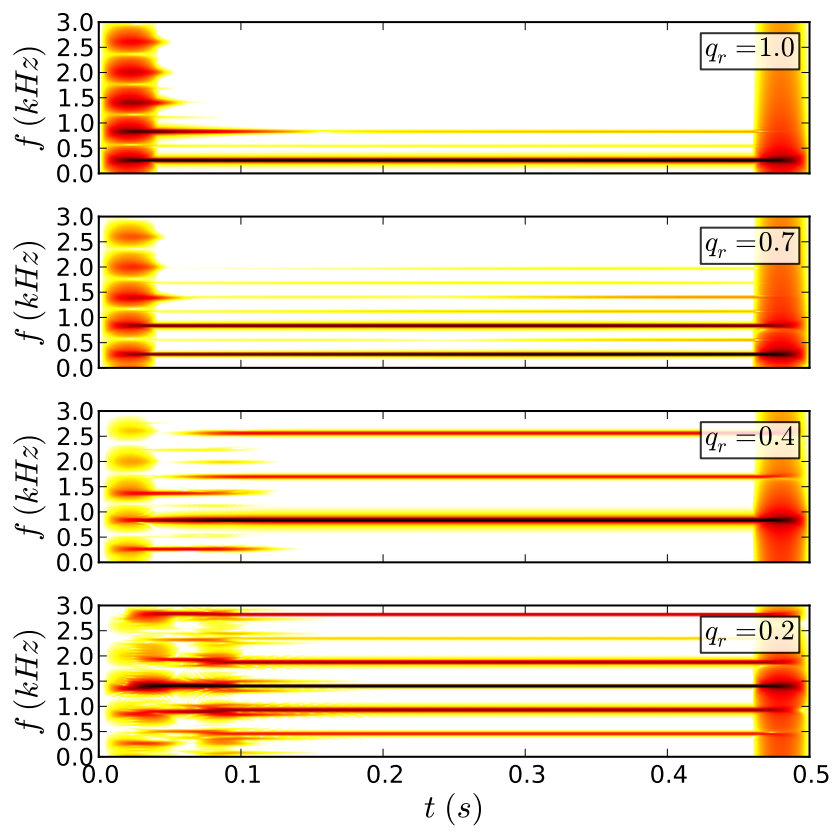

Figure 1: Spectrograms of the mouthpiece pressure simulated for various reed dampings. Cylindrical bore of length $30 \mathrm{~cm}$ and radius $7 \mathrm{~mm}$ (truncated to 12 modes), reed with effective stiffness $0.5 \mathrm{GPa} / \mathrm{m}^{2}$, natural frequency of $1500 \mathrm{~Hz}$ and equilibrium aperture of $4 \mathrm{~mm}^{2}$. The mouth pressure is a smooth step ( $\mathcal{C}^{2}$ continuous) from 0 to $780 \mathrm{~Pa}$, with an attack time of $0.5 \mathrm{~ms}$ starting at $t=5 \mathrm{~ms}$. 


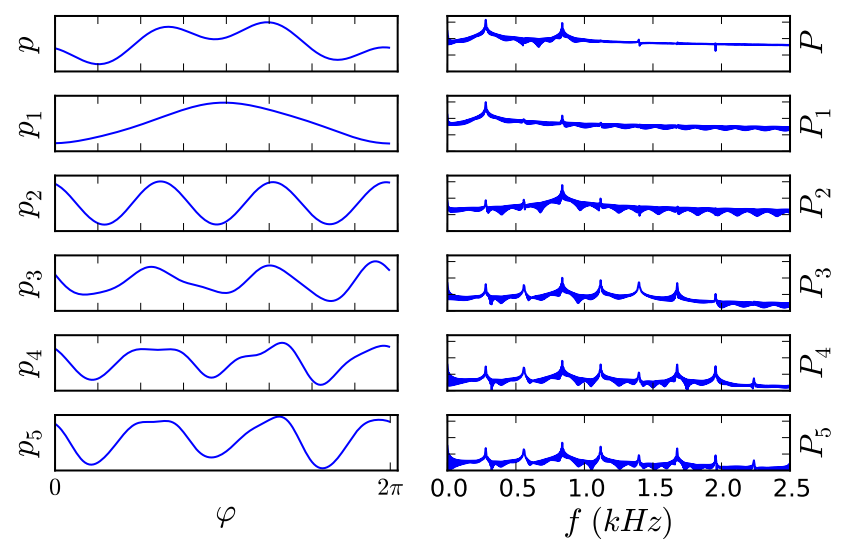

Figure 2: Steady-state waveforms (left column, one period, normalized amplitude) and spectra (right column, common axis limits and $40 \mathrm{~dB}$ between ticks) for the pressure signal (top) and its components (below). Same configuration as in Fig. 1 with $q_{r}=0.7$.

terparts. Considering the terms of the modal expansion

$$
P_{n}(\omega)=\left(\frac{C_{n}}{j \omega-s_{n}}+\frac{C_{n}^{*}}{j \omega-s_{n}^{*}}\right) U(\omega),
$$

that, if $\Re\left(s_{n} C_{n}^{*}\right)=0$, simplify to

$$
P_{n}(\omega)=\frac{Z_{n}}{1+j Q_{n}\left(\frac{\omega}{\omega_{n}}-\frac{\omega_{n}}{\omega}\right)} U(\omega),
$$

it appear to behave as a 2nd order bandpass filter bank around each of the resonances, enhancing the spectral content of the flow rate in their respective bands but not fully discarding the rest of the spectrum.

\subsubsection{Starting transients}

An important characteristic of the simulated sounds lies in the starting transient, and the manner the oscillation grows towards the steady-state [49]. Figs. 3 and 4 exhibit different behaviours for two mouth pressure dynamics. The first one, for a slow rise of the excitation, corresponds to a configuration where the lowest acoustic resonance is the destabilizing mode, growing almost exponentially. The nonlinear coupling then progressively generates harmonics. For quicker rise of the mouth pressure (cf Fig. 4), there is a distinction between the first four components, called master components in preliminary studies [42], and the next ones, called slave components. While the former grow monotonically, the latter decay from high values, like a damped impulse response, before being amplified again to reach their steady-state amplitude by non-linear coupling. Note also on the spectrogram that the first few even harmonics (the ones below the reed resonance frequency) only appear once the steady state is reached. This behaviour can be understood looking at the complex modes of the coupled system. Table 1 shows the eigenvalues of the jacobian matrix of the coupled system evaluated at the static regime for a mouth pressure equal to $1708 \mathrm{kPa}$. The first four complex eigenvalues are unstable (positive real parts) and have frequencies (the imaginary parts) close to the first four acoustic resonance frequencies, while the next ones are damped (negative real parts). The quick establishment of the mouth pressure allows the excitation of the bore resonance on a wide spectrum, providing some initial energy to the upper components. As these latter coincide with damped modes of the coupled system, their amplitudes decay. At last, the non-linear coupling reorganises the spectrum balance, amplifying the higher harmonics to reach the steady-state regime. In the case of the slow rise of the mouth pressure (Fig. 3), there is no initial excitation of the higher resonance and no such an initial decay phase.
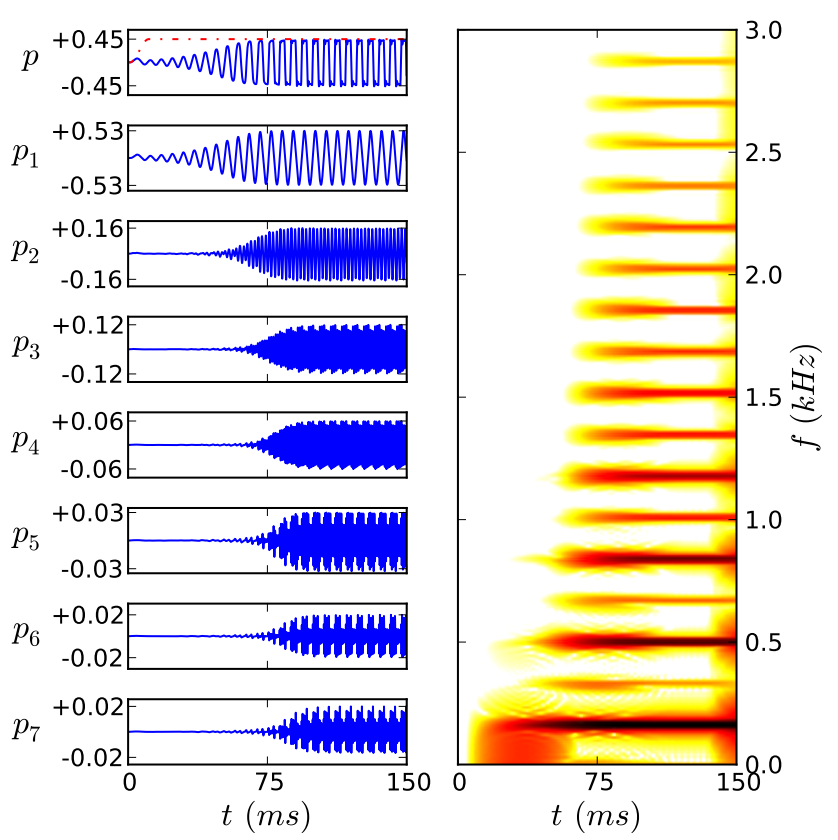

Figure 3: Time-domain representation (left column, normalised by the static beating reed pressure) and spectrogram (right column) of the mouthpiece pressure (on top), and its components (below) for a slow mouth pressure rise (attack time $10 \mathrm{~ms}$ from 0 to $1708 \mathrm{~Pa}$ ). Cylindrical bore of length $50 \mathrm{~cm}$ and radius $7 \mathrm{~mm}$ (truncated to 8 modes), reed with effective stiffness $0.5 \mathrm{GPa} / \mathrm{m}^{2}$, natural frequency of $1500 \mathrm{~Hz}$ with damping 0.4 , and equilibrium aperture of $7 \mathrm{~mm}^{2}$. Red dash-dotted line on first plot is the mouth pressure (colour online).

However, when simulating the attack transient with a measured mouth pressure (such as the one shown on Fig. 5), the components do not exhibit trivial behaviours like the ones exposed above (see Fig. 6). While the first component $p_{1}$ grows monotonically, the higher ones ( $p_{2}$ to $p_{7}$ ) follow a 

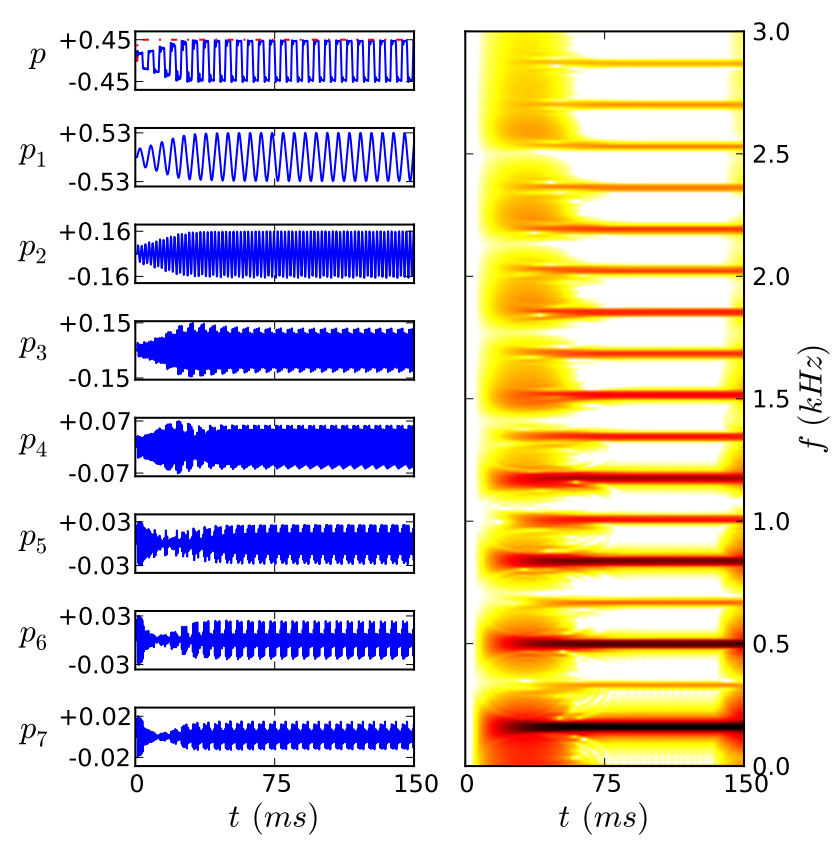

Figure 4: Fast mouth pressure rise (attack time 1/44.1 ms from 0 to $1708 \mathrm{~Pa}$ ). Same representation and system as in Fig. 3

more irregular pattern, with oscillating envelopes before stabilization (at about $t=0.11 \mathrm{~s}$ ). Another interesting point is the amplitude modulation of the higher components ( $p_{4}$ and above) at the same rate as the oscillation. Their amplitudes differ according to whether the mouthpiece pressure is close to the mouth pressure or not, i.e. whether the reed channel is closed or wide open. However the acoustic pressure $p$ is periodic. This is yet another manifestation of the difference between modal expansion and spectral analysis, and that the higher components act as slave components while the first components are the main actors of the sound

\begin{tabular}{|c|c|c|c|}
\hline \multicolumn{2}{|c|}{$\begin{array}{r}\text { Acoustic/reed } \\
\text { poles }(/ 2 \pi)\end{array}$} & \multicolumn{2}{|c|}{$\begin{array}{r}\text { Jacobian (complex) } \\
\text { eigenvalues }(/ 2 \pi)\end{array}$} \\
\hline-3.03 & $\pm 170.0 j$ & 8.44 & $\pm 168.7 j$ \\
\hline-5.28 & $\pm 513.9 j$ & 8.78 & $\pm 508.7 j$ \\
\hline-6.84 & $\pm 858.6 j$ & 12.34 & $\pm 844.8 j$ \\
\hline-8.10 & $\pm 1203.5 j$ & 12.07 & $\pm 1169.0 \mathrm{j}$ \\
\hline-9.19 & $\pm 1548.6 j$ & -16.90 & $\pm 1491.9 \mathrm{j}$ \\
\hline (reed) -300 & $\pm 1469.7 j$ & -404.45 & $\pm 1657.8 j$ \\
\hline-10.17 & $\pm 1893.8 j$ & -47.53 & $\pm 1859.3 j$ \\
\hline-11.06 & $\pm 2239.1 j$ & -44.16 & $\pm 2221.8 j$ \\
\hline-11.88 & $\pm 2584.5 j$ & -39.14 & $\pm 2572.0 \mathrm{j}$ \\
\hline
\end{tabular}

Table 1: Comparison of acoustic and mechanic poles (left column) and complex eigenvalues of the jacobian matrix of the coupled system (right column). Same system as in Fig. 3. production. Signal theory provides another interpretation of these results. Considering Eq. (19), the pressure components appear as bandpass filtered versions of the acoustic flow. Due to the losses within the bore, the higher the order of the components, the wider the filter. Assuming the non-linear coupling leads to a quite extended spectral content of the flow rate, the spectrum of a higher component $p_{n}$ may contain a limited number of significant harmonics of the oscillation frequency $\omega_{\text {osc }}$ in the vicinity of the corresponding resonance frequency $\omega_{n}$. Denoting carrier the harmonic $m \omega_{\text {osc }}$ that is the closest to the modal frequency $\left(m \omega_{o s c} \sim \omega_{n}\right)$ and side bands the others, the spectrum $P_{n}(\omega)$ can be analysed as the amplitude modulation of the carrier by a signal that has an almost discrete spectrum, i.e. a periodic signal with angular frequency $\omega_{\text {osc }}$. Depending on the spectrum of the flow rate and on the component considered, the modulating signal may take simple waveforms like square or sawtooth waves as visible on components $p_{5}$ and $p_{6}$ of Fig. 6 . Such an interpretation might be useful to propose a synthesis signal model based on the addition of periodic amplitude modulated components.

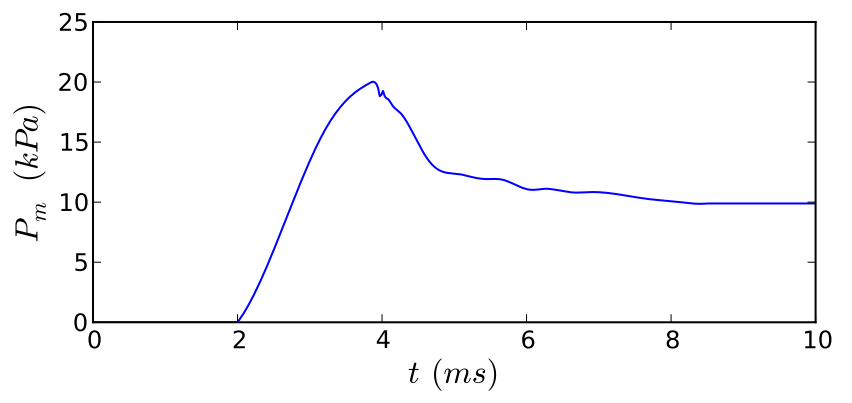

Figure 5: Measured mouth pressure (see [50] for acquisition details.)

\subsubsection{Influence of the integrator}

The different ODE integrators listed in Sec. 3 have been tested considering the same system than above, with smooth steps of the mouth pressure from 0 to various values (from the oscillation threshold to the static beating reed pressure) with $1 \mathrm{~s}$ duration. Measurements of the computational cost have been performed on a laptop (single $2.0 \mathrm{GHz}$ Pentium processor). The Euler method (with oversampling to ensure stability) has a fixed and particularly high cost (about $800 \mathrm{~s}$ for a $1 \mathrm{~s}$-simulation), while other schemes can integrate the problem up to 30 times faster due to their ability to increase the step size (LSODA and VODE) or to only evaluate a few intermediate points (Runge-Kutta methods). For a reference taken on the LSODA solver, Runge-Kutta methods are about 20\% slower due to the fact that they do not re-use previous steps, and VODE is 


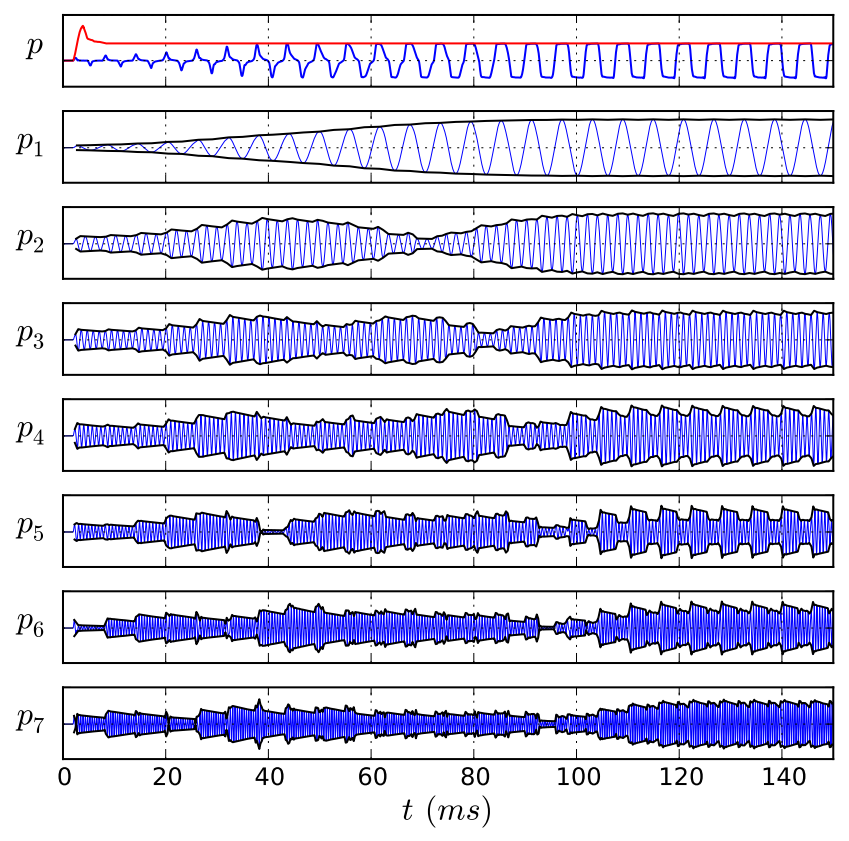

Figure 6: Dimensionless pressure signal (blue thin curves), and components (signal in blue, envelop in black) with the measured mouth pressure (red on top axes) of Fig. 5. Same system as in Fig. 3.

$40 \%$ faster (mainly because it does not check stiffness, while LSODA does). When the problem has singular points (for example, when the reed beats), the discrepancy between the Euler method and the others reduces as the latter reduce the step size to correctly handle the singularity. Concerning the accuracy of the result, the oscillation frequency during the steady state has been estimated with various methods (FFT-based spectral methods and the YIN algorithm [51]) leading to a standard deviation below the estimator resolution $(\Delta f=0.01 \mathrm{~Hz}$, i.e. 0.1 cents in the vicinity of the oscillation frequency $168.1 \mathrm{~Hz}$ ) for all but the Euler method (1 cents).

The system is then simulated for a linear rise of the mouth pressure from 0 to the static beating-reed pressure (8.54 kPa here) over a $5 \mathrm{~s}$ duration. For this dynamic bifurcation diagram, similar timings are obtained (considering LSODA as the reference, Euler is $+280 \%$ slower, RungeKutta $5(4)+27 \%$, Runge-Kutta $8(5,3)+50 \%$ slower, and VODE is $45 \%$ faster). Fig. 7 highlights one of the consequences of the numerical resolution of the problem defined in the continuous time domain. Numerical dispersion is introduced, modifying the acoustic impedance and the mechanic transfer function. This has consequences both on the dynamic bifurcation (see Fig. 7a) and on the oscillation frequency above the oscillation threshold (as shown in Fig. 7b). The latter exhibits similar results as those obtained for the steady state oscillation: all but the Euler method show an excellent agreement once the transient is over. The influ- ence of the integration method on the dynamic bifurcation is quite dramatic, leading to a $10 \%$ dispersion of the estimation threshold. It is important to understand that the solvers are suitable for different purposes. This case requires a solver that is able to cope with stiff problems. In fact, at the oscillation threshold, one of the coupled system's eigenvalues crosses zero, the system temporarily has a very large (if not infinite) characteristic timescale, being then described as stiff. Concerning this example, the VODE solver has been forced to use the AM method (designed for non-stiff problems) and only the LSODA solver handles correctly the bifurcation.

However simpler methods are still useful when analysing the evolutions away from bifurcation values. In fact, Fig. 8

(a)
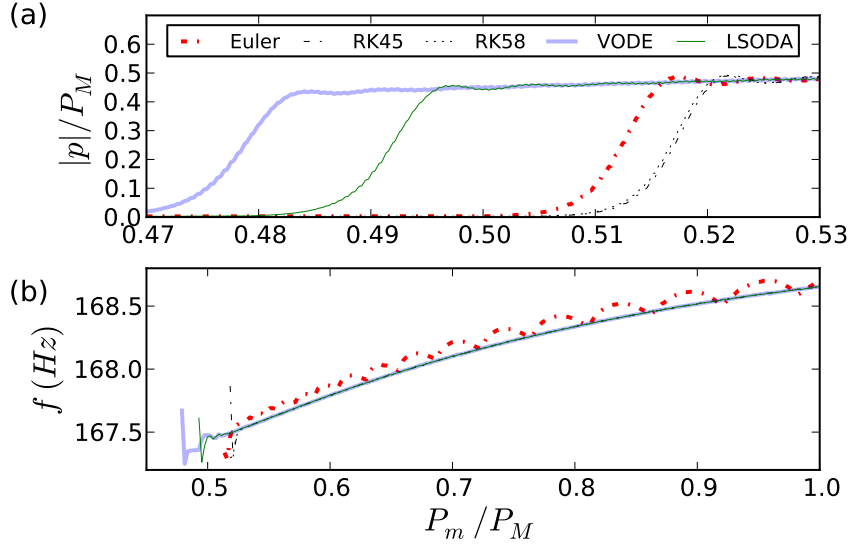

Figure 7: (a) Pressure amplitudes near oscillation threshold, and (b) oscillation frequency for a linear variation of the mouth pressure from 0 to the static beating reed pressure $P_{M}=8.5 \mathrm{kPa}$ in the $[0,5 \mathrm{~s}]$ time range. Same system as in Fig. 3.

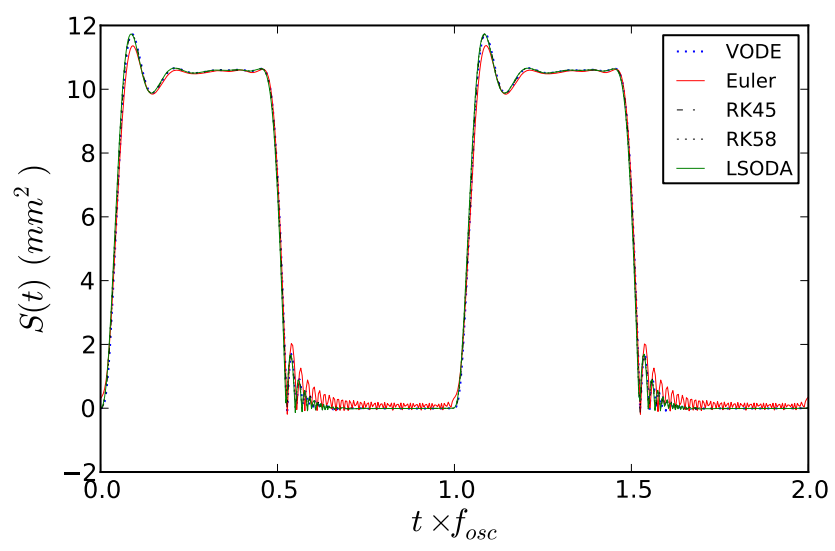

Figure 8: Same simulations as Fig. 7: waveforms of the reed opening when the mouth pressure reaches $90 \%$ of the static beating reed pressure $P_{M}=8.5 \mathrm{kPa}$. Same system as in Fig. 3. 
focuses on the part of the previous simulations where the linearly increasing mouth pressure equals $90 \%$ of the static beating reed pressure (with time scaling due to Euler method's result having a different oscillation frequency, and time shifting to synchronize the rising fronts). Except for the Euler method which lead to slightly different results (weaker damping of the oscillations), all the results show a good agreement on the waveforms of the reed opening although the integrations had to deal with a quite complex path including the bifurcation and nonlinearities such as the beating reed regime.

\subsection{Brass instruments}

\subsubsection{Modal expansion of a measured input impedance}

Simulation can also be performed using measured impedances. Fig 9 shows an input impedance curve for the open-valves position of a Yamaha YTR1335 trumpet. The process leading to an acceptable modal representation of this impedance will be discussed in a future paper. However 12 modes are considered hereafter (see values in Table 2), with a parametrisation error less than $2 \%$ on complex values (visible above $1300 \mathrm{~Hz}$ on Fig. 9).

\subsubsection{Transient slurs}

The first simulation is performed with a natural frequency $\omega_{r}$ of the lips linearly decreasing from $1 \mathrm{kHz}$ to $100 \mathrm{~Hz}$ and a lip damping $q_{r}$ kept constant at value 0.1 leading to a lip stiffness $K_{r}$ equal to $0.8 \mathrm{GPa} / \mathrm{m}^{2}$. Concerning the control parameters, the opening area at rest $h_{0}$ was set at $10 \mathrm{~mm}^{2}$, while the mouth pressure $P_{m}$ was a smooth step $\left(\mathcal{C}^{1}\right.$ profile) from 0 to $20 \mathrm{kPa}$ with a $1 \mathrm{~ms}$ transient. The LSODA integrator was used, taking advantage from its automatic switches between nonstiff and stiff methods to handle difficulties at the changes of playing regime. The simulation is
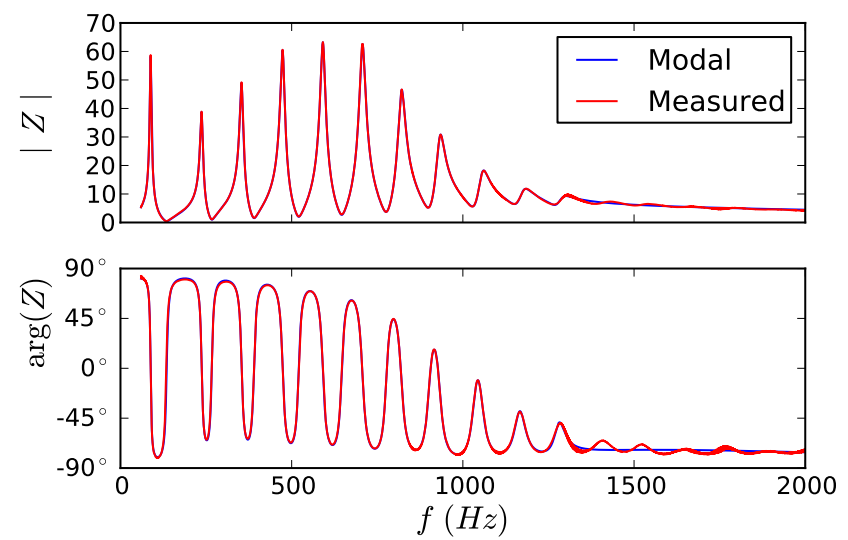

Figure 9: Dimensionless impedance (and its modal representation) of the trumpet with all valves opened (12 modes).

\begin{tabular}{r|c|c|c} 
& Natural frequency & Quality factor & Amplitude \\
$n$ & $f_{n}$ & $Q_{n}$ & $Z_{n}$ \\
\hline 1 & $88.16 \mathrm{~Hz}$ & 18.6 & 58.12 \\
2 & $237.16 \mathrm{~Hz}$ & 29.0 & 38.20 \\
3 & $353.89 \mathrm{~Hz}$ & 33.5 & 48.22 \\
4 & $473.80 \mathrm{~Hz}$ & 35.7 & 59.72 \\
5 & $591.29 \mathrm{~Hz}$ & 38.0 & 62.53 \\
6 & $706.70 \mathrm{~Hz}$ & 38.0 & 61.88 \\
7 & $820.30 \mathrm{~Hz}$ & 37.7 & 45.12 \\
8 & $932.05 \mathrm{~Hz}$ & 36.9 & 27.80 \\
9 & $1054.77 \mathrm{~Hz}$ & 34.9 & 14.12 \\
10 & $1173.35 \mathrm{~Hz}$ & 31.1 & 6.54 \\
11 & $1284.21 \mathrm{~Hz}$ & 32.3 & 3.39 \\
12 & $1397.63 \mathrm{~Hz}$ & 1.1 & 1.46 \\
\hline
\end{tabular}

Table 2: Numerical values given for the equivalent modal representation (see Eq. (19)).

programmed quite as simply as shown in Listing 1 . Further explanations on the programming interface are available in the documentation of the package.

Fig. 10 shows the evolution of the spectral content of the simulated mouthpiece pressure. The lip reed interacts with the respective acoustic resonances, selecting the one that has a natural frequency just above its own. However this behaviour shows some latency, as the change of regime does not occur exactly at the instant where the lip resonance crosses a lower bore resonance. This may be related to results in Refs. [52, 53], but also to the existence of simultaneously stable regimes, or the dynamical bifurcation characteristics as reported in Ref. [54].

Listing 1: Example of simulation of slurred transients using a measured input impedance

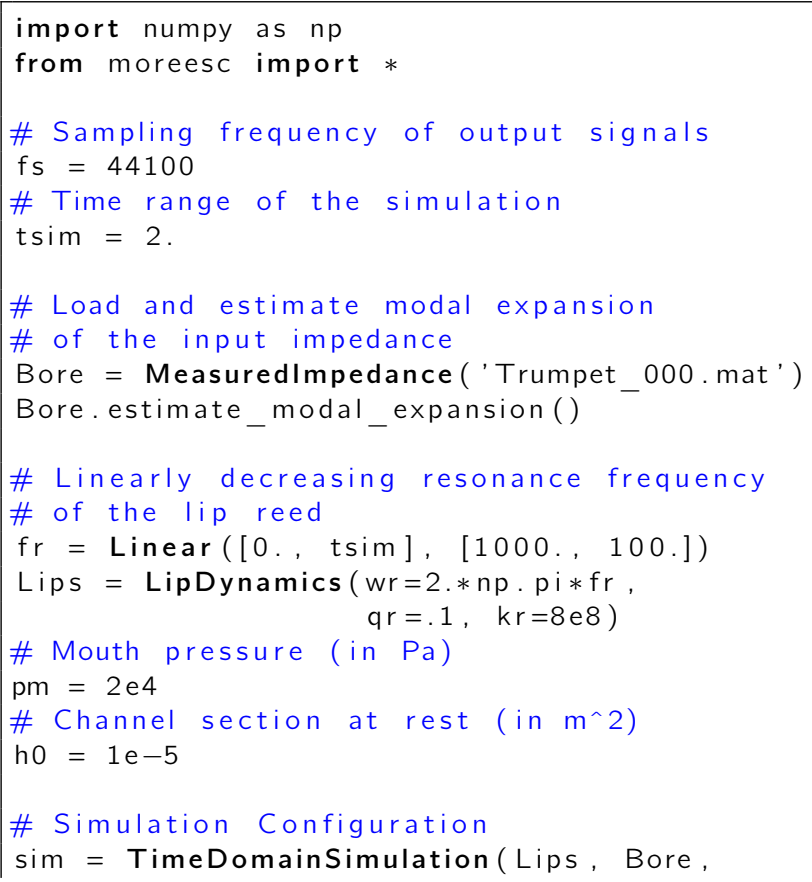




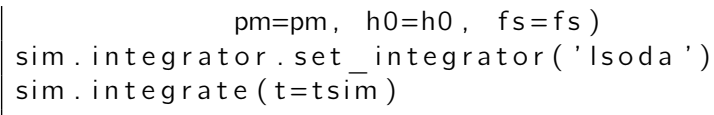

Fig. 11a evidences the difference between downward and upward slurred transients. As a complement of the above spectrogram, the downward slur exhibits registers centering on the configuration where the lips resonance frequency matches one of the acoustic one. This compares to results from linear stability analysis (see Ref. [52], and Fig. 5 in [53] for low values of $\omega_{r}$ ). On the contrary, upward slurs behave quite differently, the regime changes appear when the mechanic resonance frequency overcomes one of the bore's frequencies, showing a similarity with what occurs for high values of $\omega_{r}$ in Fig. 5 in [53]. This offset becomes even more significant when observing the deviation of the instantaneous frequency to the notes of the tempered scale that are meant to be played for these configurations (see Fig. 11b). Oscillations obtained by downward slurs appear to be flatter than those resulting from upward slurs. This corresponds to the sensation of the musician when practising three-notes slurs (up and down, and vice versa). It is also interesting to notice that upward slurs lead to excessively sharp notes (except for $A \# 4$ ) requiring the musician to adopt some strategy to adjust the intonation.

\subsubsection{Wah-wah effect}

The last example illustrates the ability to define a timevarying acoustic resonator. This is exemplified through the use of a rubber plunger mute on the same trumpet. Impedance measurements are analysed to extract the poles and residues for several positions of the mute from closing of the bell to wide opening. These values are then arranged to

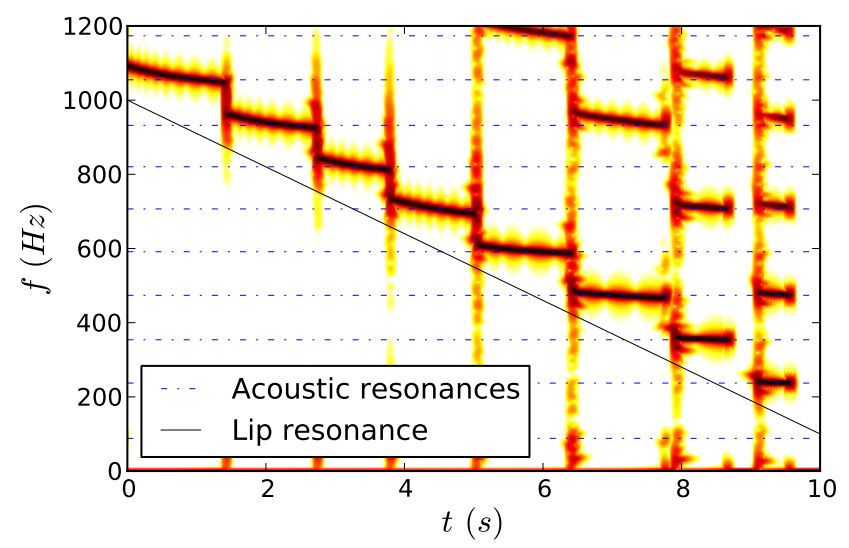

Figure 10: Spectrogram of the mouthpiece pressure. Lips $q_{r}=0.1, K_{r}=0.8 \mathrm{GPa} / \mathrm{m}^{2}, \omega_{r}$ linearly decreasing from $1 \mathrm{kHz}$ to $100 \mathrm{~Hz}$ (black line). Resonator described in Table 2 (acoustic resonances as dash-dotted lines). The mouth pressure is a smooth step from 0 to $20 \mathrm{kPa}$, and $h_{0}=10 \mathrm{~mm}^{2}$.
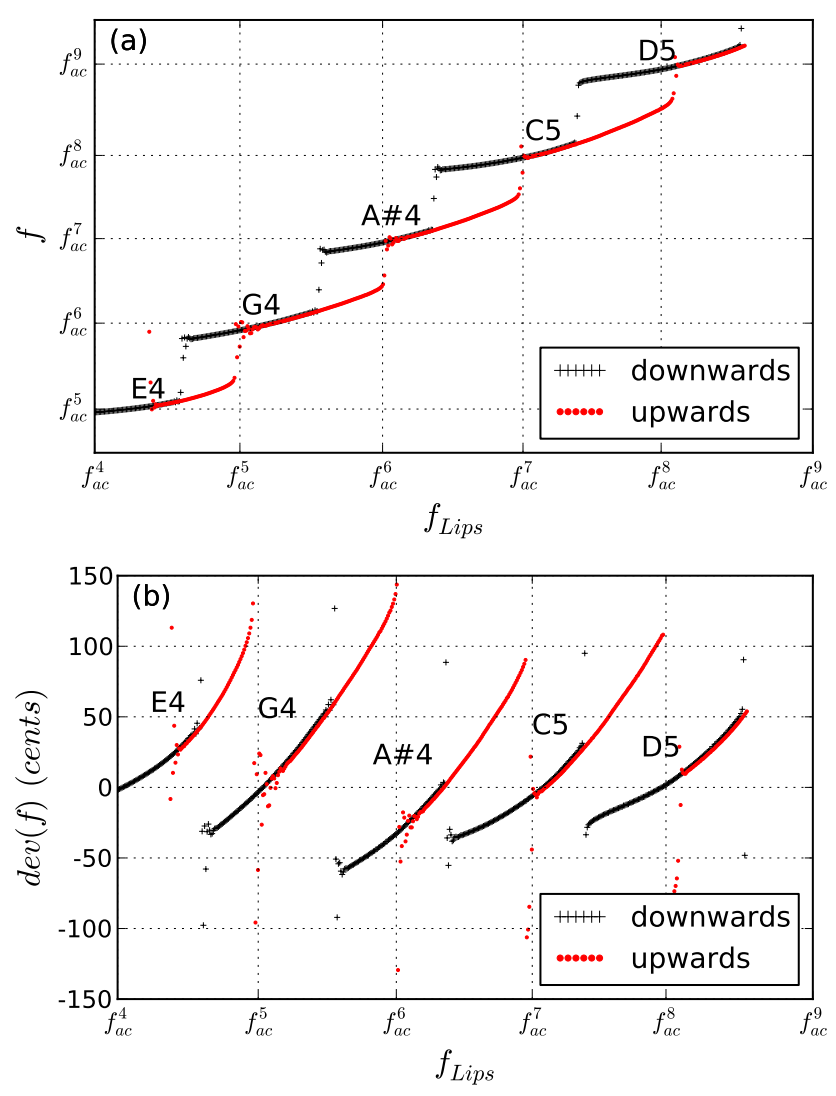

Figure 11: (a)Instantaneous frequency $f$ and (b) deviation of $f$ to respective tempered scale note $f_{T S}$ with respect to the lips buzzing frequency $f_{\text {Lips }}$ for downward and upward slurred transients. Written pitch for the Bb trumpet.

create a time-varying input impedance, so that it simulates a sequence of closings/openings of the bell. Practically, for each pole-residue pair, a cubic Bézier curve is defined as an interpolation between closed bell and open bell values with two intermediate positions [55]. The impedance thus can evolve continuously during the time range of the simulation. Programmatically, this is done in a few lines of code only. In Fig. 12, the variations of the resonance frequencies $\left(\omega_{n}\right)$ show deviations from the muteless case less than 30 cents.

The estimation of the instantaneous frequency leads to deviation of the same order of magnitude as the acoustic resonance frequencies. The evolution of the fundamental amplitude and of the spectral centroïd frequency (based on the partial amplitudes of the mouthpiece pressure) show (see Fig. 13) a stronger impact, that can possibly lead to perceptible variations of the loudness and the timbre, characteristics of the so-called Wah-wah effect. 


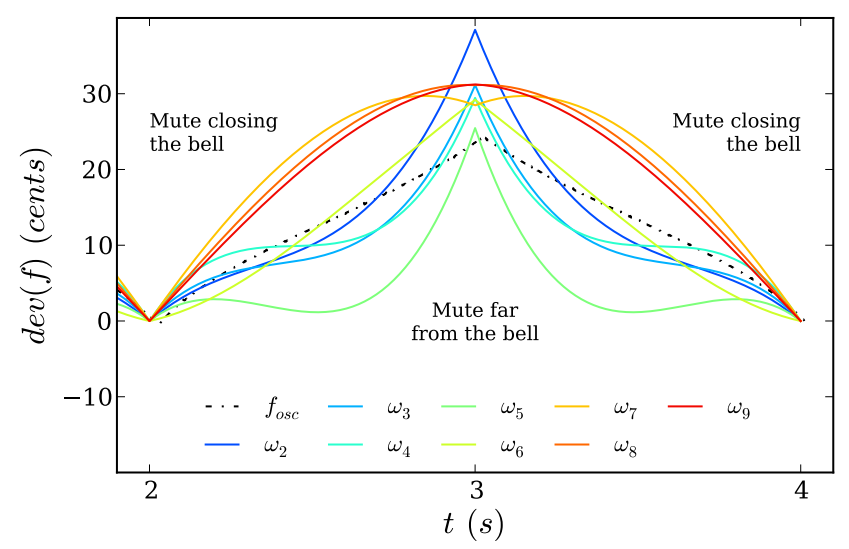

Figure 12: Evolution of the acoustic resonance frequency (solid curves) and of the oscillation instantaneous frequency (dashed-dotted curve) when toggling on and off the plunger mute. Lips with natural frequency $500 \mathrm{~Hz}$, damping 0.1 and stiffness $800 \mathrm{GPa} / \mathrm{m}^{2}$ with opening area equal to $10 \mathrm{~mm}^{2}$ at rest. Mouth pressure constant $(20 \mathrm{kPa})$.
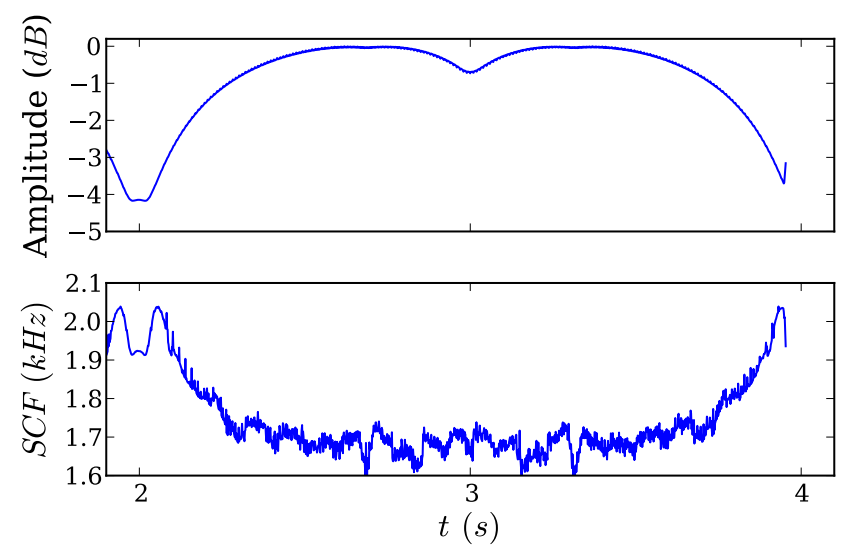

Figure 13: Amplitude of the fundamental of the mouthpiece pressure (top) and spectral centroïd frequency (bottom). Same simulation as in Fig. 12.

\section{Limitations}

From the perspective of physical-based modelling of musical instruments, the main weakness of the MoReeSC software lies in a unique localised interaction. In fact, both the acoustic and the mechanic components are modelled as seen by the coupling jet: the bore is reduced to its input impedance, and the reed to its opening section. While quite common in the musical acoustics community, this formulation has two drawbacks. At first glance, it masks the spatial dimension of the bore, and thus makes the evaluation of the sound radiation of the instrument difficult. However, if the spatial modes $\varphi_{n}(x)$ related to the poles of the acoustic resonator are known, it is possible to evaluate the pressure and/or the volume velocity at any point within the bore. Further- more, the modal expansion may be applied to the transfer function between the input flow rate and an external pressure (as provided by some impedance measurement device) so that the radiated pressure can be computed, providing a more realistic timbre than the one of the mouthpiece pressure. Notwithstanding it seems quite uneasy to consider non-linear behaviour of the acoustic resonator, such as nonlinear propagation (at high sound levels) or non-linear losses at the open toneholes.

Another concern lies in technical aspects. Recent work [54] has brought into focus the influence of the numerical precision used in simulations. While investigating the bifurcation delay between static and dynamic oscillation threshold, they note that the use of the common double precision (64 bits storage corresponding to 15 significant decimal digits precision) may be insufficient to numerically study this problem, depending of the rate of change of the control parameters. The MoReeSC simulation framework is based on solvers that explicitly use double precision, at least when considering variable step-size methods. Further investigation is needed to determine if this numerical problem also impacts other dynamical characteristics.

\section{Discussion and perspectives}

This paper presents the MoReeSC software, a framework available to the musical acoustics community, with source code, binaries and documentation at www.moreesc.lma. cnrs-mrs.fr, where examples described in this paper can also be found. The interface has been designed so that the users only have to manipulate objects and concepts very close to physical models. It alleviates the pain of building discrete time models by only requiring a continuous time formulation where the coefficients have direct physical interpretations, saving thus scientist time but still enabling him to determine whether the focus is to be put on speed or on precision by easily changing the ODE solver. Modularity is also a key word, as little effort is also required to implement new coupling relations. The software also offers tools for a quite straightforward use of measurements.

Relying on mature libraries for computation, postprocessing and visualisation (odepack, Python and its scientific ecosystem: NumPy, Scipy, Matplotlib), the MoReeSC software fits into a recent effort to provide freely distributed tools useful for researchers in music technologies and particularly in musical acoustics, amongst which are AUBIO (audio signal analysis, from the Centre for Digital Music, Queen Mary Univ. London), WIAT (Wind Instrument Acoustic Toolkit from the Computational Acoustic Modeling Laboratory, McGill Univ.) or SMS (spectral modelling synthesis, from the MTG, Pompeu Fabre Univ.). In addition to these topical softwares, it benefits from the general-purpose ones that may be of some interest in the musical acoustics community. For example, MoReeSC software is able to share the 
continuous-time domain formulation with tools like PyDSTool which, amongst other features, offers continuation and bifurcation analysis tools. Neuroscience has recently seen such an effort be successful (the NeuralEnsemble effort), by promoting code sharing and re-use across laboratories, preferring open-source tools to closed-source commercial products. A similar trend is occurring in the astronomy field.

Concerning the MoReeSC software, the future work will be devoted to improve the interface with existing experimental and numerical tools. First the modal expansion is the key point between impedance measurement devices and MoReeSC . A further study will focus on proper ways to handle measured impedances and how the modal representation has to be completed to be suitable both for continuous and discrete time formulation. The results of the simulations can also be used to explore the model reduction possibilities.

\section{Acknowledgements}

This work was supported by the French ANR research projects CONSONNES and CAGIMA. The authors wish to thank Whitney Coyle and Sami Karkar for helpful remarks and valuable comments on the manuscript.

\section{References}

[1] A. H. Benade, Fundamentals of Musical Acoustics, ch. The Woodwinds: I, pp. 430-464. New-York: Oxford Univ. Press, 1976.

[2] F. Silva, J. Kergomard, C. Vergez, and J. Gilbert, "Interaction of reed and acoustic resonator in clarinet-like systems," J. Acous. Soc. Am., vol. 124, pp. 32843295, 2008.

[3] J. Gilbert, J. Kergomard, and E. Ngoya, "Calculation of the steady-state oscillations of a clarinet using the harmonic balance technique," J. Acous. Soc. Am., vol. 86, no. 1, pp. 35-41, 1989.

[4] S. Farner, C. Vergez, J. Kergomard, and A. Lizée Deblevid, "Contribution to harmonic balance calculations of self-sustained periodic oscillations with focus on single-reed instruments," J. Acous. Soc. Am., vol. 119, pp. 1794-1804, 2006.

[5] B. Ricaud, P. Guillemain, J. Kergomard, F. Silva, and C. Vergez, "Behavior of reed woodwind instruments around the oscillation threshold," Acta Acustica united with Acustica, vol. 95, no. 4, pp. 733-743, 2009.

[6] S. Karkar, C. Vergez, and B. Cochelin, "Oscillation threshold of a clarinet model: A numerical continu- ation approach," J. Acous. Soc. Am., vol. 131, no. 1, pp. 698-707, 2012.

[7] W. E. Worman, Self-sustained nonlinear oscillations of medium amplitude in clarinet-like systems. PhD thesis, Case Western Reserve University, 1971.

[8] J. Kergomard, S. Ollivier, and J. Gilbert, "Calculation of the spectrum of self-sustained oscillators using a variable truncation method: Application to cylindrical reed instruments," Acta Acustica united with Acustica, vol. 86, pp. 685-703, 2000.

[9] V. Välimäki, J. Pakarinen, C. Erkut, and M. Karjalainen, "Discrete-time modelling of musical instruments," Rep. Prog. Phys., vol. 69, pp. 1-78, 2006.

[10] J. O. Smith, "Virtual acoustic musical instruments: Review and update," J. New Music Res., vol. 33, no. 4, pp. 283-304, 2004.

[11] S. E. Stewart and W. J. Strong, "Functional model of a simplified clarinet," J. Acous. Soc. Am., vol. 68, pp. 109-120, 1980.

[12] S. Bilbao, Numerical Sound Synthesis: Finite Difference Schemes and Simulation in Musical Acoustics. John Wiley \& Sons, 2009.

[13] J.-L. Florens and C. Cadoz, "Synthesizing the physical model: Modeling and simulating the instrumental universe," in Representations of Musical Signals (G. De Poli, A. Piccialli, and C. Roads, eds.), pp. 227268, Cambridge, MA: MIT Press, 1991.

[14] M. van Walstijn and M. Campbell, "Discrete-time modeling of woodwind instrument bores using wave variables," J. Acous. Soc. Am., vol. 113, no. 1, pp. 575585, 2003.

[15] G. P. Scavone, An Acoustic Analysis of Single-Reed Woodwind Instruments, with an Emphasis on Design and Performance Issues and Digital Waveguide Modeling Techniques. PhD thesis, Stanford University, 1997.

[16] P. Guillemain, J. Kergomard, and T. Voinier, "Realtime synthesis of clarinet-like instruments using digital impedance models," J. Acous. Soc. Am., vol. 118, no. 1, pp. 483-494, 2005.

[17] J. Martinez, J. Agulló, and S. Cardona, "Conical bores. part ii: Multiconvolution," J. Acous. Soc. Am., vol. 84, no. 5, pp. 1620-1627, 1988.

[18] B. Gazengel, J. Gilbert, and N. Amir, "Time domain simulation of single reed wind instrument. from the measured input impedance to the synthesis signal. where are the traps?," Acta Acustica, vol. 3, pp. 445472, 1995. 
[19] J. Antunes, M. G. Tafasca, and L. L. Henrique, "Simulation of the bowed-string dynamics-part 1- a nonlinear modal approach," in Actes du 5ème congrès français d'acoustique (SFA, ed.), p. 285-288, 2000.

[20] R. E. Caussé, J. Bensoam, and N. Ellis, "Modalys, a physical modeling synthesizer: More than twenty years of researches, developments, and musical uses," J. Acous. Soc. Am., vol. 130, no. 4, pp. 2365, 2011.

[21] A. Barjau and V. Gibiat, "Study of woodwind-like systems through nonlinear differential equations. part ii. real geometry," J. Acous. Soc. Am., vol. 102, no. 5, pp. 3032-3037, 1997.

[22] H. von Helmholtz, On the Sensations of Tone, ch. App. VII, p. 5388. New York: Dover Publications, Inc., 1954

[23] R. T. Schumacher, "Ab initio calculations of the oscillation of a clarinet," Acustica, vol. 48, pp. 71-85, 1981.

[24] M. E. Mclntyre, R. T. Schumacher, and J. Woodhouse, "On the oscillations of musical instruments," J. Acous. Soc. Am., vol. 74, pp. 1325-1345, 1983

[25] T. A. Wilson and G. S. Beavers, "Operating modes of the clarinet," J. Acous. Soc. Am., vol. 56, pp. 653-658, 1974.

[26] J. O. Smith, Introduction to Digital Filters with Audio Applications. Music signal processing series, W3K Publishing, 2006.

[27] S. Ollivier, Contribution à l'étude des oscillations des instruments à vent à anche simple (Contribution to the study of oscillations in single reed instruments). PhD thesis, Univ. Maine, Le Mans, 2002.

[28] F. Avanzini and M. van Walstijn, "Modelling the mechanical response of the reed-mouthpiece-lip system of a clarinet. part i. a one-dimensional distributed model," Acta Acustica united with Acustica, vol. 90, pp. 537547, 2004.

[29] B. Gazengel, T. Guimezanes, J.-P. Dalmont, J. B. Doc, S. Fagart, and Y. Léveillé, "Experimental investigation of the influence of the mechanical characteristics of the lip on the vibrations of the single reed," in ISMA, (Barcelona, Spain), 2007.

[30] M. J. Newton, M. Campbell, and J. Gilbert, "Mechanical response measurements of real and artificial brass players lips," J. Acous. Soc. Am., vol. 123, no. 1, pp. 14, 2008.

[31] F. Silva, V. Debut, J. Kergomard, C. Vergez, A. Lizée Deblevid, and P. Guillemain, "Simulation of single reed instruments oscillations based on modal decomposition of bore and reed dynamics," in ICA, (Madrid, Spain), 2007.

[32] A. Chaigne and J. Kergomard, Acoustique des instruments de musique. BELIN, 2008. ISBN 978-2-70113970-8.

[33] A. S. Phani and J. Woodhouse, "Viscous damping identification in linear vibration," J. Sound Vib., vol. 303, no. 3-5, pp. 475-500, 2007.

[34] D.-M. Tran, "Component mode synthesis methods using partial interface modes: Application to tuned and mistuned structures with cyclic symmetry," Computers and Structures, vol. 87, no. 17-18, pp. 1141-1153, 2009.

[35] P. Guillemain and F. Silva, "De l'utilisation de la décomposition modale pour la synthèse sonore temps réel : écueils et solutions (using modal expansion in realtime sound synthesis)," in 10ème Congrès Français d'Acoustique, (Lyon, France), 2010.

[36] A. Hirschberg, , "Aeroacoustics of wind instruments," in Mechanics of Musical Instruments (A. Hirschberg, J. Kergomard, and G. Weinreich, eds.), pp.291-369, CISM Courses and Lectures No.355, (Wien - New York), Springer-Verlag, 1995.

[37] A. R. da Silva, G. P. Scavone, and M. van Walstijn, "Numerical simulations of fluid-structure interactions in single-reed mouthpieces," J. Acous. Soc. Am., vol. 122, pp. 1798-1809, 2007.

[38] A. Almeida, C. Vergez, and R. Caussé, "Quasi-static non-linear characteristics of double-reed instruments," J. Acous. Soc. Am., vol. 121, no. 1, pp. 536-546, 2007.

[39] G. Fant, Acoustic Theory of Speech Production. D A C S R Series, Mouton De Gruyter, 1970.

[40] G. C. J. Hofmans, G. Groot, M. Ranucci, G. Graziani, and A. Hirschberg, "Unsteady flow through in-vitro models of the glottis," J. Acous. Soc. Am., vol. 113, pp. $1658-1675,2003$

[41] C. J. Nederveen, Acoustical aspects of woodwind instruments. DeKalb: Northern Illinois, 1998.

[42] V. Debut, Deux études d'un instrument de musique de type clarinette: Analyse des fréquences propres du résonateur et calcul des auto-oscillations par décomposition modale (Two studies of a clarinet-like musical instrument: analysis of the eigen frequencies and calculation of the self-sustained oscillations by modal decomposition). PhD thesis, Aix-Marseille Univ., 2004. 
[43] P. Guillemain and A. Merer, "Rôles du contrôle et du timbre dans la perception du naturel de sons de clarinette," in 10ème Congrès Français d'Acoustique, (Lyon, France), 2010.

[44] A. C. Hindmarsch, "Odepack, a systematized collection of ode solvers," IMACS Trans. Sci. Comp., vol. 1, pp. 55-64, 1983.

[45] P. N. Brown, G. D. Byrne, and A. C. Hindmarsh, "Vode: a variable-coefficient ode solver," SIAM J. Sci. Stat. Comput., vol. 10, no. 5, pp. 1038-1051, 1989.

[46] E. Jones, T. Oliphant, P. Peterson, et al., "Scipy: Open source scientific tools for python," 2001.

[47] J. R. Dormand and P. J. Prince, "A family of embedded runge-kutta formulae," Journal of Computational and Applied Mathematics, vol. 6, no. 1, pp. $19-26,1980$.

[48] F. Silva, Émergence des auto-oscillations dans un instrument de musique à anche simple (Emergence of self sustained oscillations in single reed woodwind instrument). PhD thesis, Aix-Marseille Univ., 2009.

[49] J. M. Grey, "Multidimensional perceptual scaling of musical timbres," J. Acous. Soc. Am., vol. 61, pp. 12701277, 1977.

[50] P. Guillemain, C. Vergez, D. Ferrand, and A. Farcy, "An instrumented saxophone mouthpiece and its use to understand how an experienced musician play," Acta Acustica united with Acustica, vol. 96, no. 4, pp. 622634, 2010.

[51] A. de Cheveigne and H. Kawahara, "Yin, a fundamental frequency estimator for speech and music," J. Acous. Soc. Am., vol. 111, no. 4, pp. 1917-1930, 2002.

[52] J. S. Cullen, J. Gilbert, and M. Campbell, "Brass instruments: Linear stability analysis and experiments with an artificial mouth," Acta Acustica united with Acustica, vol. 86, no. 4, pp. 704-724, 2000.

[53] F. Silva, J. Kergomard, and C. Vergez, "Oscillation thresholds for "striking outwards" reeds coupled to a resonator," in ISMA, (Barcelona, Spain), 2007.

[54] B. Bergeot, A. Almeida, C. Vergez, and B. Gazengel, "Prediction of the dynamic oscillation threshold in a clarinet model with a linearly increasing blowing pressure," to appear in Nonlinear Dynamics, 2013.

[55] P. Guillemain and J. Terroir, "Dynamic simulation of note transitions in reed instruments: application to the clarinet and the saxophone," in Comp. Music Modeling and Retrieval, (Pisa, Italy), 2005. 